\title{
Lire Zola pour apprendre la fraternité
}

Read Zola to learn about brotherhood

\section{Agnès Perrin-Doucey and Jacques-Louis Perrin}

\section{(2) OpenEdition}

\section{Journals}

Electronic version

URL: http://journals.openedition.org/trema/5722

DOI: 10.4000/trema.5722

ISSN: 2107-0997

\section{Publisher}

Faculté d'Éducation de l'université de Montpellier

\section{Electronic reference}

Agnès Perrin-Doucey and Jacques-Louis Perrin, «Lire Zola pour apprendre la fraternité », Tréma [Online], 53 | 2020, Online since 01 March 2020, connection on 10 December 2020. URL : http:// journals.openedition.org/trema/5722 ; DOI : https://doi.org/10.4000/trema.5722

This text was automatically generated on 10 December 2020 .

Trema 


\title{
Lire Zola pour apprendre la fraternité
}

\author{
Read Zola to learn about brotherhood
}

Agnès Perrin-Doucey and Jacques-Louis Perrin

\section{Introduction}

On connait la destinée chaotique de l'acception laïque du mot fraternité dans notre histoire depuis la Révolution. L'apport politique tardif, peut-être d'origine maçonnique, ne s'imposera qu'en 1848 grâce à Louis Blanc. Il subira ensuite une longue éclipse avant de réapparaitre le 14 juillet 1880 sur les frontons de toutes les Institutions publiques, associé aux deux mots liberté et égalité pour former le triptyque adopté définitivement comme symbole officiel de la République. Ce mot souvent remis en cause par nombre de penseurs de la IIIe République qui lui préféraient la notion de solidarité, moins connotée spirituellement et moins exigeante aussi bien socialement que philosophiquement, reste toujours un symbole éminemment ambigu, au «signifiant [particulièrement] flottant» (Gritti, 1983, p. 211) compte tenu de la variété des référents culturels auquel il se rattache. Sa réalisation semble quelque peu gênante car elle apparait comme une obligation morale plutôt que juridique, contrairement aux principes de liberté et d'égalité1.

2 Les définitions des dictionnaires elles-mêmes sont rendues ambigües par le foisonnement des acceptions qu'il engage. L'idée de «lien» reste le dénominateur commun proposé par le Trésor de la Langue Française Informatisé, lien de sang, lien d'amitié, lien tribal ou racial, lien d'apprentissage ou de conviction : autant de relations communautaires qui s'opposent, à priori, à la connotation universaliste que veut lui conférer la pensée révolutionnaire depuis le 18e siècle. Le Robert (1995), quant à lui, définit la fraternité comme "un lien existant entre les hommes considérés comme membres de la famille humaine ", donc "frères ", comme le signale l'origine latine du mot: frater. Ce dictionnaire met aussi l'accent sur le fait que la fraternité est «l'expérience de la profondeur et du mouvement», une formule qui nous parait 
particulièrement intéressante et sur laquelle nous serons amenés à revenir dans la suite de notre réflexion. Ces quelques rappels historiques et linguistiques expliquent peut-être pourquoi nos contemporains, capables de définir assez facilement les notions de « liberté " et d'" égalité ", sont assez déconcertés lorsqu'il s'agit de préciser le sens qu'ils donnent au mot fraternité, auquel ils préfèrent le plus souvent l'idée de "solidarité ". Les élèves, et même parfois les étudiants, sont eux aussi souvent hésitants, surtout quand les référents littéraires, historiques et culturels fondamentaux font défaut.

3 C'est pourquoi il nous semble plus que jamais indispensable, surtout dans le contexte éducatif actuel qui nous conduit à revisiter de telles valeurs universelles (MEN, 2015a), d'interroger les enjeux historiques d'un concept qui irrigue l'attachement à la République et nourrit la formation du citoyen. Or, parmi tant d'autres actions éducatives propres à mieux cerner la notion et à la faire vivre, le recours à des textes patrimoniaux ou classiques permet à la fois d'éclairer de façon précise la notion et de restituer ses sens profonds. Nous considérons en effet, à l'appui des travaux de Véronique Larrivé (2014), que la fiction littéraire est apte à véhiculer des « émotions [...] empathiques" qui, rencontrant les "émotions personnelles des lecteurs", toucheront leur sensibilité, surtout s'ils parviennent à articuler une dialectique entre les dimensions participative et distanciée de la lecture (Dufays, 2002).

4 Abdennour Bidar (2015), s'interrogeant sur l'urgence de fraternité à laquelle notre société lui parait soumise, affirme « la fraternité s'apprend. On ne nait pas fraternel, on le devient»(p.72). Si l'on accepte cette position, alors comment faire apprendre la fraternité ? quels travaux proposer aux élèves ? Comment approcher simplement et de façon conjointe les expressions littéraires, historiques et culturelles de la fraternité pour favoriser une transmission positive de valeurs destinées à fonder une société ?

5 Nous postulons ici que la lecture littéraire peut jouer un rôle déterminant, notamment quand elle recourt aux romans historiques, ou plus généralement à des fictions ancrées dans un moment emblématique de notre histoire. Pour le montrer, après avoir interrogé l'idée de fraternité et cerné quelques-uns des enjeux de sa transmission à la lumière des recherches philosophiques et littéraires récentes, nous illustrerons notre réflexion par une lecture axiologique d'extraits de La Fortune des Rougon (Zola, 1871) premier roman de la série des Rougon-Macquart, pensé pour une classe de Première. Ainsi, nous tenterons de mettre en évidence la manière dont une fiction littéraire peut participer à la formation morale et civique en contribuant à l'éducation à la fraternité du futur citoyen qu'est l'élève.

\section{Choisir la fraternité : de l'espoir au plaidoyer}

« Il fait trop froid dans la vie en Occident » affirme Abdennour Bidar (2015, p. 85) en interrogeant le rôle que la fraternité peut jouer dans notre société. Par là-même, il reprend la réflexion engagée dès 2004 par Catherine Chalier. Associant, dès le titre de l'ouvrage, la fraternité à " un espoir en clair-obscur ", elle évoque, dans une réflexion mâtinée d'étonnement, les paradoxes de la nature humaine que le principe de fraternité révèle. Pour elle, « les misères sidérantes provoquées par les ruptures du lien humain " n'empêchent pas «le témoignage de fraternité [de se faire] encore parfois entendre, à contretemps presque toujours » (12). Elle montre alors comment «l'unicité insubstituable» des relations interindividuelles, ces «relation[s] d'unique à 
unique donne[nt] sens à toutes les autres relations humaines»(Ibid.). De ce fait, la fraternité, qu'elle considère comme "irréductiblement plurielle» et "jamais fusionnelle», semble précéder les "différents types de communauté ou de société pensés ou imaginés par les hommes pour vivre ensemble sans se détruire. » (13)

C'est bien cette réflexion qu'Abdennour Bidar reprend dans un plaidoyer destiné à inciter ses contemporains à prendre "la décision de fraternité" $(2015$, p. 67) pour sauver les dimensions universelles qui fondent l'humanité. Là encore, les deux philosophes s'accordent pour reconnaitre que la «fraternité dépend de l'éclat très éphémère de l'amour sur nos vies » dont « la tonalité immémoriale surprend chacun(e) et l'incite à passer sur la rive du "me voici" du frère ou de la sœur " (Chalier, 2004, p. 153). Cependant, dans un monde gangréné par l'individualisme, le quant à soi, le moi-je, l'expérience de la fraternité se réduit comme peau de chagrin, se heurte au désir de considérer l'autre à l'aune de soi-même comme un " frère qui me ressemble » (Bidar, op. cit.: 123). Le principe de fraternité, dans son acception républicaine, incite au contraire à la recherche d'un lien d'empathie, voire de sympathie avec l'altérité, c'està-dire avec l'autre qui diffère de moi par ses appartenances (identitaire, religieuse, civilisationnelle, etc.) et qui se lie à moi par l'universalité de son humanité. A. Bidar considère que le public jeune qui n'a pas encore pu éprouver ce principe reste particulièrement fragile. Il signale ainsi, se référant au contexte troublé des attentats qui ont endeuillé Paris en janvier 2015, la perte de certaines valeurs républicaines fondamentales :

Nos valeurs - dignité de l'être humain, liberté, égalité, fraternité, solidarité, laïcité, mixité - ont besoin d'être réapprises par notre société tout entière, et pas seulement rappelées à quelques musulmans radicaux! [...] Notre nation tout entière a oublié d'en assurer l'enseignement, la transmission, les moyens d'un partage vivant ( $\mathrm{p}$. 42).

Pour Catherine Chalier, l'espoir de fraternité trouve son sens dans l'intelligence de l'immémorial qui éclaire le langage humain. Elle le perçoit notamment dans la force manifestée par la résistance des Hommes face aux tentatives de déshumanisation. Dans ces contextes, les individus se saisissent de la puissance fondatrice de l'être ensemble permis par le lien fraternel pour réaffirmer leur appartenance humaine.

Pour Abdennour Bidar, la transmission de la fraternité implique d'oser enfin parler du sacré tous ensemble. Le mot a de quoi surprendre, surtout quand on le reprend pour interroger les relations qui s'imposent au 21e siècle entre fraternité et éducation dans le cadre laïque que définit l'école publique française. Dès lors il faut se demander de quel sacré il peut bien s'agir.

Il faut remplacer la guerre des sacrés contradictoires par la paix du sacré partagé. Le sacré est pour un être humain ce qui lui tient le plus à cœur, ce pour quoi il serait prêt à donner sa vie, à se "sacrifier » [...] N'ayons plus peur de ce mot de sacré! N'ayons plus peur de parler entre nous du sacré. [...] Il est temps de prendre enfin le risque non seulement d'en parler mais de proposer un sacré partageable un sacré qui n'empêche pas les uns de croire en tel dieu et les autres de se déclarer athées, agnostiques ou indifférents. Un sacré qui n'entre en concurrence ni avec les religions ni avec l'athéisme. Personnellement, je ne vois que la fraternité qui remplisse ces critères. C'est en effet une valeur transversale, universelle, que l'on trouve dans tous les héritages d'Orient et d'Occident, aussi bien dans les sagesses religieuses que dans les morales profanes (Ibid., p. 62-64). lien à l'autre comme miroir de soi-même, lien susceptible d'exclure, selon Catherine 
Chalier, « l'idée d'une fraternité possible avec le dissemblable, avec celui ou celle qui ne partagerait ni identité, ni essence commune avec soi» (2004, p.123-124). Dans son plaidoyer, Abdennour Bidar pense que le principe de fraternité, à condition qu'on veuille bien lui conférer une valeur sacrale, est à même de transcender l'essentialisme des appartenances idéologiques et de rapprocher les individus par et pour leur humanité. Ce principe ainsi pensé nous ramène donc à l'essence de notre humanité, à son universalité qui nous rapproche, même dans la dissemblance, en nous gardant de la suprématie de la loi du plus fort. Selon le philosophe, historiquement, la France joue un rôle primordial dans cette réalisation axiologique parce qu'elle incarne encore pour les autres pays le symbolique du « peuple qui s'est libéré par la Révolution » (Ibid., 64).

11 Cependant, considérant que la chaleur humaine, cet "éclat éphémère de l'amour " (Chalier, 2004, p. 153), a déserté nos espaces sociaux, en France notamment, nous pouvons alors nous demander avec Abdennour Bidar: "Où est notre capacité à fraterniser au-delà du cercle restreint de nos proches? Où est notre capacité à nous mettre à la place de l'autre ? [...] Où est l'élan du cœur dans tout cela ? (p. 62-65)». De telles analyses impliquent évidemment d'interroger l'idée et les conditions de transmission. En effet, ces deux philosophes ouvrent, semble-t-il, une voie éducative étroite et exigeante pour développer l'espoir d'une société fraternelle. Elle semble résonner comme une obligation pour la formation morale et civique des élèves. Or, une question demeure : comment éduquer à cette fraternité qui accueille « celui ou celle qui ne partag[e] ni identité, ni essence commune avec soi " (Chalier, p. 124), comment aborder la dimension sacrée du lien fraternel dont parle Abdennour Bidar?

La fiction littéraire, parce qu'elle est mimesis, nous semble être un moyen puissant, à condition d'en proposer une lecture alliant émotion et raison, de construire cette expérience fondatrice de l'altérité que requiert à priori la transmission des valeurs fraternelles.

\section{Lire des romans pour apprendre à accueillir l'autre dans sa dissemblance}

13 L'enseignement littéraire, s'il est conçu dans une approche sémioticienne trop oublieuse des dimensions anthropologique et axiologique portées par la diégèse des textes, ne permet pas de penser les lectures faites en classe comme autant d'expériences symboliques du monde. Dans ces conditions, on ne favorise pas une lecture critique, car c'est l'enseignant qui détermine à priori l'interprétation du texte, sans prendre en compte les ressources axiologiques mobilisées par les élèves et les valeurs portées par le passage lu, ne sont pas mises en discussion. Dès lors, il serait inenvisageable de lui attribuer des fonctions éducatives et émancipatrices pour la formation du citoyen, car on prendrait le risque de l'engager dans l'édification d'un discours moraliste pouvant aller jusqu'à justifier «les stéréotypes moraux les plus éculés » (Michel, 2016, p. 292). Cependant, aujourd'hui, cette conception de la relation entre éthique et littérature est remise en cause au profit d'une approche anthropologique et axiologique qui repense la place du lecteur (Todorov, 2007). Notre propos se situera donc dans une conception rénovée de l'enseignement littéraire portée depuis la fin du 20e siècle par les travaux des didacticiens de la littérature autour de la notion de sujet-lecteur (Langlade \& Rouxel, 2004). En effet, à l'aube de ce nouveau millénaire, une partie de la critique (Compagnon, 2007-2008; Citton, 2007; Todorov, 
2007) et nombre de didacticiens (Louichon \& Perrin-Doucey, 2016; Mas, 2017; Rouvière, 2019; Louichon \& Sauvaire, 2019) semblent vouloir renouer avec les dimensions émancipatrices de la lecture quand elle est pensée pour un lecteur singulier apte à nouer un dialogue fécond entre émotion et raisonnement.

Selon Antoine Compagnon (2009), il est effectivement nécessaire de retrouver les anciens pouvoirs de la littérature pour qu'elle nous permette de «devenir qui nous sommes ». Rejetant l'idée selon laquelle « la perplexité est la seule morale littéraire » (1998, p. 392), il plaide désormais pour que la conception et les études littéraires amorcent un tournant éthique.

Il serait risible que les littéraires renoncent à la défense et illustration de la littérature au moment où d'autres disciplines la retrouvent avec empressement, en particulier l'histoire culturelle et la philosophie morale (p. 61-62).

Le critique montre d'une part comment les historiens des mentalités, proches de l'Ecole des Annales (comme Maurice Agulhon, Alain Corbin ou Pierre Nora), s'intéressent à la transmission par le livre et par la lecture, et d'autre part que « la philosophie morale analytique et la théorie des émotions investissent de plus en plus les textes littéraires ». Rappelant que la lecture des romans a irrigué «l'initiation morale en Occident depuis deux siècles", il affirme que, selon ces philosophes, la littérature "peut aider au développement de notre personnalité ».

Elle permet d'accéder à une expérience sensible et à une connaissance morale qu'il serait difficile, voire impossible d'acquérir dans les traités des philosophes. Elle contribue donc de manière irremplaçable à l'éthique pratique comme à l'éthique spéculative (p. 63).

\section{Antoine Compagnon conclut enfin que la lecture littéraire}

offre un moyen [...] de préserver et de transmettre l'expérience des autres, ceux qui sont éloignés de nous dans l'espace et le temps, ou qui diffèrent de nous par les conditions de leur vie (Ibid.).

17 C'est bien parce que la lecture de la fiction littéraire contribue à organiser une expérience des autres, que son approche en classe de littérature peut favoriser une réflexion sur la fraternité. Il s'agit effectivement de faire appel à l'empathie du lecteur, empathie ressentie grâce à la dimension participative de la lecture, et empathie analysée dans la relation que les personnages développent dans la diégèse. Véronique Larrivé (2015), interrogeant les mécanismes émotionnels actionnés dans la lecture de la fiction, évoque, à l'appui des travaux de Keith Oatley, une similitude entre l'empathie que peut déclencher une projection dans la fiction et les relations sociales. Ainsi, selon elle :

Lorsqu'il imagine le personnage en train d'agir, le lecteur se met donc lui-même virtuellement en action, en simulant au niveau neuronal les gestes du protagoniste dont il lit l'histoire et en activant pour ce faire son propre répertoire moteur. C'est en partie ainsi qu'il comprend l'action du personnage. Et par le même phénomène, le lecteur de fiction qui imagine un personnage en train de souffrir éprouve virtuellement la même souffrance, ce qui lui permet de comprendre, même de façon peu subtile à cette étape, ce que le personnage ressent. C'est donc aussi en utilisant son propre répertoire émotionnel que le lecteur peut identifier les émotions du personnage (p. 160).

18 Ainsi, par l'immersion fictionnelle, le lecteur installe des relations interindividuelles avec un autre, protagoniste d'une fiction, dans une simulation sensible et imaginative instillant peut-être cette «complicité pour rien » que requiert l'idée de fraternité « irréductiblement plurielle » évoquée par Catherine Chalier (2006, p. 13). 
19 Vincent Jouve, quant à lui, dans sa Poétique des valeurs constate que « Le texte peut [...] faire accepter, le temps d'une lecture, des valeurs non établies, voire déstabilisantes » (p.33), d'autant que désir et valeur, comme Philippe Hamon (1984) et lui-même le remarquent, sont toujours étroitement associées. Le lecteur, se projetant dans cette forme d'altérité, partage avec le protagoniste les obstacles rencontrés qui ne font que renforcer « le pouvoir d'attraction de la quête et instituent en valeur la lutte contre les forces du mal et le refus de l'indifférence et de la démission» (p. 34). Pour V. Jouve, c'est parce qu'un lien de sympathie unit lecteur et personnage « qu'il devient d'autant plus difficile de ne pas le soutenir dans son entreprise ».

Le jeu affectif qui s'élabore dans cette relation engage plus aisément le lecteur dans l'acceptation des foyers idéologiques portés par le texte. De cette façon, affirme le critique, le récit romanesque y gagne une force de persuasion qui devient un remarquable « moyen d'enseignement et de prédication » (p. 34).

21 S'appuyant sur les travaux récents des critiques comme des didacticiens, Nicolas Rouvière (2019) tente de circonscrire la question dans sa globalité. S'intéressant à la fois au texte lui-même, comme au lecteur et à son activité, il affirme que la lecture axiologique s'appuie sur « six composantes » qui peuvent contribuer « à conduire à une réflexivité éthique sur les valeurs qu'on mobilise dans la lecture» (p. 32). Ces six composantes sont problématisées par le chercheur en trois points précis :

- certains éléments proviennent du texte lui-même comme c'est le cas avec la composante « sémiotique : poétique et rhétorique des valeurs » (p. 33);

- d'autres éléments proviennent de l'interaction texte et lecteur comme avec la composante " génétique » qui se demande «d'où viennent les valeurs en jeu dans le texte et sa lecture » (p. 37) ;

- d'autres éléments proviennent enfin plutôt du lecteur et de l'activité de lecture. C'est le cas des composantes: «d'engagement éthique et empathique dans la lecture» (p.32), " appréciative, du jugement de gout à l'attribution critériée d'une valeur à l'œuvre littéraire » (p. 35), (pré)philosophique de formation de la pensée (p. 39) et «(pré)civique : quel rapport à la vérité dans la communauté interprétative ?» (p. 41).

Avec cette ébauche de théorisation didactique, $N$. Rouvière espère pouvoir apporter une clarification notionnelle favorisant un enseignement de la littérature qui questionne les valeurs et non l'inverse. Il ouvre ainsi plus largement le champ didactique de la formation éthique réflexive des élèves et atteste qu'à certaines conditions, la lecture et l'étude des fictions littéraires peuvent contribuer à interroger les valeurs. Dès lors, elles doivent permettre d'éprouver - au sens de mise à l'épreuve - le caractère sacré de la fraternité, tel que l'entend Abdennour Bidar. Mais cette relation d'unique à unique qu'instaure l'appropriation singulière du texte par chaque sujetlecteur ne peut donner sens à l'ensemble des relations humaines que si elles sont partagées et discutées au sein de la communauté des lecteurs dans des débats tout à la fois « empathiques » et « raisonnés » (Louichon \& Perrin-Doucey, 2017). Il s'agit donc de faire appel aux "réactions axiologiques " (Vibert, 2011) des sujets-lecteurs et de les confronter, sans nier l'émotion qui les a provoquées, pour installer, le temps du cours,

cette complicité pour rien qui, parfois, vient interrompre le cours toujours intéressé des affaires et des relations humaines, pour y jeter une clarté fragile mais indestructible, la fraternité viendrait rappeler que la réalité amère et dure, impitoyable et cruelle, en laquelle s'enferment si souvent les humains, n'est ni première, ni ultime (Chalier : 13). 
Ainsi, la communauté de lecteurs que devrait instaurer pour un temps le cours de littérature, s'il engage un dialogue entre empathie et raison, pourrait approcher symboliquement l'idée de fraternité, et l'ériger au rang de sacré partageable. Quand le texte de fiction étudié s'y prête notamment par ses composantes sémiologiques (PerrinDoucey, 2019b), les élèves pourront approcher, par l'expérience fictionnelle, «les conséquences politiques d'un certain oubli de la fraternité dans les sociétés modernes soucieuses de promouvoir l'individu comme source essentielle de tous ce qui a valeur et dignité [...]» (Ibid. : 95). Le dispositif didactique à mettre en œuvre pour y parvenir, doit prendre en compte l'ensemble de ces perspectives et articuler dans les pratiques effectives plusieurs des "composantes" (Rouvière, 2019, p. 44) énoncées ci-dessus. C'est ce que nous voudrions exemplifier maintenant à partir de l'élaboration d'un dispositif didactique organisant une lecture anthropologique, axiologique et comparative de deux passages de la Fortune des Rougon (Zola, 1871)

\section{Mettre les élèves à l'épreuve de la fraternité}

La visée exploratoire de notre propos ne se construira pas sur une analyse de pratiques effectives. Elle se situe plutôt en amont, dans une réflexion sur les moyens à mettre en œuvre pour construire un dispositif didactique de lecture littéraire centré sur la manière dont s'exprime le concept de fraternité dans les textes. Nous faisons donc le choix ici d'interroger la lecture littéraire des experts. Ce préalable à la construction du projet de séance interroge, avant toute chose, les ressources portées par les dimensions sémiotiques, rhétoriques et axiologiques des textes. C'est aussi l'acte d'un sujet-lecteur qui transcende sa lecture personnelle pour chercher les leviers de sens inhérents à la construction didactique ultérieure.

Cette étape de la préparation d'un cours est peu souvent présentée dans les travaux des didacticiens centrés le plus souvent sur le rôle et la parole du lecteur. Pourtant, «le pilotage du cours est intimement lié à la préparation de celui-ci» (Bucheton, 2020 : 1938). Il nous parait donc nécessaire d'éclairer aussi le cheminement professionnel par des lectures expertes, surtout dans une visée axiologique. À l'issue de cette étude littéraire, nous proposerons quelques pistes pour la réalisation du dispositif en luimême, notamment en nous demandant par quels moyens mettre la problématique à la portée des élèves. En effet, l'objet d'enseignement que nous visons dans le cadre de cette étude de textes vise à dépasser l'explication du texte, qu'elle soit analytique ou linéaire, pour tenter de "mettre à l'épreuve» de la fraternité nos élèves en "questionnant la littérature par les valeurs " (Ibid. : 45). Dès lors, l'étude sémiotique poétique et rhétorique de l'enseignant, comme les ressources qu'il mobilise durant sa lecture singulière, permettront de mieux saisir les outils nécessaires au pilotage d'une lecture scolaire centrée sur les valeurs.

\section{Le choix de l'ouvrage}

Nous avons choisi de travailler à partir du roman de Zola La Fortune des Rougon, car l'ouvrage met en jeu les valeurs qui nous préoccupent dans des situations narratives que tout oppose. Par ailleurs, malgré la distance temporelle, le récit peut faciliter le processus identificatoire des élèves de lycée, les deux héros du livre : Silvère et Miette étant de très jeunes adolescents. Enfin, le roman évoque une révolte violente menée 
par des insurgés du sud de la France contre le coup d'état de Louis Napoléon Bonaparte en 1851. La thématique, au-delà des connaissances historiques qu'elle requiert et favorise, peut organiser des effets d'échos, hier comme aujourd'hui, avec les préoccupations du jeune public, confronté lui-même, directement ou indirectement par les médias, à telle ou telle manifestation sociale ou politique. L'expérience collective de la révolte est fondatrice pour la construction du citoyen en devenir qu'est l'élève. Elle rencontrera, si le pilotage de la séance le permet, l'expérience personnelle des sujetslecteurs dans la classe. Dès lors, comme le suggère N. Rouvière, les composantes sémiotique et génétique de l'œuvre peuvent participer à créer un effet de miroir réfléchissant entre l'expérience qu'implique la lecture et le vécu personnel de chaque élève. Une telle œuvre, à condition d'en ménager une lecture axiologique qui fait entrer en résonance le lu et le vécu de la communauté de lecteurs constituée par la classe, parait donc être à même de contribuer à développer une conscience (pré)civique et (pré)philosophique autour de l'idée de fraternité.

Pour ce faire, nous proposons d'extraire deux passages du roman qui seront travaillés dans une dimension comparative nécessaire au développement d'un débat favorisant une articulation entre empathie et raison.

\section{Le premier extrait : la fraternité en marche}

Dans le premier passage extrait du chapitre 1, Silvère, jeune héros du roman, regarde passer, subjugué, une troupe d'insurgés qui se révoltent contre le coup d'état de Louis Napoléon Bonaparte durant la « semaine sanglante » du 7 au 14 décembre 1851. Trentedeux départements sont en état de siège et vingt se soulèvent.

Au début de cette œuvre, Zola met en scène une petite armée qui, partie de quelques villages du sud de la France, et chantant La Marseillaise, gagne Plassans, microcosme provincial réagissant de façon mécanique aux évènements qui se déroulent dans le macrocosme parisien, offrant donc une image à la fois inversée et grotesque de la vraie vie politique.

Silvère rejoint bientôt ces insurgés, ainsi que Miette, son amour de treize ans, qui deviendra l'égérie du groupe. Très vite, la scène prend une dimension mythique, métamorphosant l'Histoire, en entrelaçant trois types de fiction: le roman d'amour entre deux jeunes adolescents, le roman historique qui évoque le coup d'État de 1851 dans une petite ville du Sud, et le roman à la fois généalogique (itinéraire d'une famille) et cosmogonique (par le rappel de la faute originelle, du meurtre fondateur: "Ci-gist Marie morte "), qui va servir de socle aux Rougon-Macquart. Ainsi, Zola parvient à faire agir les trois niveaux axiologiques définis par V. Jouve : le niveau local, incarné par l'agir des personnages; le niveau idéologique global, déterminé par l'autorité énonciative d'un narrateur omniscient, et la réalité historique, entremêlée à la fiction narrative; le niveau programmatique, installé par l'outrance maitrisée dans laquelle Zola tisse les dimensions généalogiques et cosmogoniques de l'œuvre.

31 Dans le passage qui nous occupe, le lecteur assiste en effet à l'entrée théâtrale de la troupe des trois mille insurgés dans une nature somptueuse, devenant "grandiose " par l'irruption humaine. Le petit cours d'eau nommé la Viorne qui la parcourt a été grossi par les pluies et Zola file la métaphore du torrent, torrent à la fois humain et naturel. Les hommes s'identifient à la crue et la nature méridionale s'humanise, légitimant l'insurrection car il y a complémentarité et symbiose des forces humaines et 
naturelles en présence. Zola donne à voir et à entendre une vaste symphonie théâtrale ${ }^{2}$ chargée de symboles, symphonie à la fois pastorale et patriotique où l'ordre physique et l'ordre humain fusionnent et deviennent source de beauté. Un puissant mouvement la parcourt, généré par les phrases longues et segmentées, le jeu des sonorités constrictives et des assonances en [i], les verbes d'action et le jeu des temps. Après le silence initial, la métaphore du concert prolonge celle de la crue avec la montée en puissance de La Marseillaise soulignée par les hyperboles et les vastes périodes. Et bientôt, c'est un orchestre complet que nous fait entendre Zola, les voix humaines trouvant dans la vallée de la Viorne une forte caisse de résonance. Cette création poétique à fonction historique rappelle à bien des égards le tableau de Delacroix $L a$ Liberté guidant le peuple, tissant ainsi par l'intertextualité un stéréotype symbolique de fraternité imaginée par des hommes "voulant vivre ensemble sans se détruire " (Chalier, 2003, p. 13). C'est une remarquable page exaltant le sentiment de fraternité qui saisit les insurgés et les deux adolescents, unis dans la même ferveur révolutionnaire - Silvère les "appelle du nom de frères ", mot clé du passage. Cette page renvoie à des moments essentiels de notre Histoire, avec trois partenaires agissant au diapason: le jeune couple, la nature et les républicains, qui constituent alors l'unicité d'un être collectif, massif et déterminé. Autant d'images idéalisées d'une révolution rêvée dans un décor fantastique aux couleurs froides et métalliques qui, avec la présence de la lune, donnent à l'évènement une dimension romantique et wagnérienne, mystérieuse et tragique.

Vibrations, mouvement, profondeur, unité : ces impressions rappellent la définition que donne le dictionnaire Le Robert de la fraternité (« un lien existant entre les hommes considérés comme membres de la famille humaine »), impressions qui, une fois décelées par les élèves pourront être mises en discussion.

\section{Le second extrait : les « Assis»}

En contrepoint, et afin de tenter d'approcher ce qu'est le contraire de la fraternité humaine, nous pourrions proposer un extrait du même roman (La Fortune des Rougon, ch. 3, de : "L'idée de réussir » à " poussé à bout »), texte dans lequel Zola évoque une réunion des bourgeois partisans de Louis-Napoléon Bonaparte dans le salon jaune de Félicité Rougon à Plassans.

En effet, dans ce salon, tout est l'exact opposé de ce qui est décrit dans le premier texte étudié. À l'espace ouvert, naturel et vivant parcouru allègrement par des insurgés enthousiastes et unis s'oppose un lieu clos, immobile et mortifère, où les «masques figés » des bourgeois comploteurs peinent à cacher leurs appétits féroces. Le salon jaune apparait comme l'espace de la prédation, de l'alliance momentanée d'intérêts bas et divergents. Un monde de loups où l'humain s'animalise. Une société cloisonnée régie par des lois que la nature réprouve. Un lieu du pouvoir occulte qui prend, par glissement métonymique, une vie autonome et devient le centre d'une fable symbolique, simple et frappante. Par exemple, Zola évoque «les opinions du fameux salon jaune » (p. 120). À cet instant du roman, le salon jaune connote la dissimulation, le cynisme, la jalousie, la cruauté des anciens marchands d'huile. Il est un substitut du noir, perversion des vertus de l'intelligence, couleur des traitres fomentant leur complot. Et tout au long du roman, la couleur jaune s'oppose au rouge révolutionnaire, 
porteur d'idéaux de loyauté et d'honnêteté dont la jeune Miette, à l'image de l'égérie peinte par Delacroix, se fait le porte-drapeau.

Ce monde des prédateurs (des Assis, dirait Rimbaud ${ }^{3}$ !) nous est montré, dans ce deuxième extrait, à travers le regard acéré et intéressé du Docteur Pascal qui, se livrant à un bel exercice de physiognomonie, où observations scientifique et caricature se conjuguent. Le personnage compare alors le salon jaune à une "ménagerie » habitée par " une sauterelle verte ", " un crapaud [...] blême et visqueux ", " un mouton gras ", " un vieux dogue édenté », « un veau », etc. Dans cette « ménagerie », le docteur ne sera jamais à sa place, n'étant pas "vétérinaire ", comme il le lance ironiquement à sa mère Félicité !

Ce passage se construit donc, par le jeu de la focalisation interne, sur une mise en évidence des dissonances axiologiques entre l'agir de personnages avides de réussite personnelle qui peuplent le microcosme du salon jaune et la caricature que livre au lecteur ce visiteur contraint de partager cet instant au cœur du salon jaune «pour ne pas chagriner » Félicité. Ainsi, l'écriture conditionne la répulsion du lecteur, l'invitant à rejeter l'espace clos et symbolique de l'anti-fraternité.

Notons enfin, pour compléter l'étude que, dans les dernières pages de La Fortune des Rougon, on assiste à un transfert et une inversion des couleurs particulièrement intéressants. Un crépuscule jaune éclaire faiblement de ses reflets louches l'assassinat de Silvère. La couleur jaune, concentrée jusque-là dans le salon, envahit l'espace extérieur. Le triomphe des " jaunes ${ }^{4}$ » commence, mais il est à jamais éclaboussé par le sang de la répression. Les dernières pages de l'œuvre ont, à cet égard, une forte charge symbolique. N'est-ce pas une représentation allégorique de l'espoir en clair-obscur qu'incarne, pour Catherine Chalier, la fraternité?

\section{Vers l'élaboration d'un dispositif didactique}

En analysant les composantes sémiotiques et génétiques de deux extraits d'une œuvre patrimoniale, nous avons voulu montrer qu'ils constituent des supports intéressants pour notre projet. En effet, ils favorisent d'une part la pluridisciplinarité en nouant des croisements historiques et culturels que le dispositif didactique devra mettre à la portée des élèves. D'autre part, ils mettent en scène les jeux politiques qui se tissent autour de l'ambition de pouvoir et le désir d'égalité que les élèves pourront actualiser afin d'en saisir la portée universelle. Le dispositif didactique lui-même reste à construire et nous souhaitons ici apporter quelques pistes précises pour l'organiser.

Il s'agit de proposer une lecture qui implique, en même temps, la sensibilité et le raisonnement des élèves pour les conduire à penser éthiquement et civiquement à partir des deux extraits choisis. Pour ce faire, notre étude montre qu'il indispensable de proposer des éléments contextuels comme des outils favorisant l'actualisation indispensable à l'engagement du sujet-lecteur en formation.

\section{Des ressources supplémentaires}

Les élèves devront retrouver les repères historiques et culturels indispensables à la compréhension de la pensée de l'époque. Pour ce faire, il est toujours intéressant de travailler en interdisciplinarité avec le professeur d'histoire. Mais il est aussi possible 
de constituer un dossier documentaire complémentaire permettant de s'approprier les éléments contextuels.

41 Cependant, cette restitution du contexte ne suffit pas, il faut aussi conduire les élèves à penser leur propre projection dans une telle situation pour saisir les enjeux qui poussent le couple Sylvère et Miette dans l'aventure humaine qu'ils traversent au rythme de leur expérience amoureuse. Pour cela, il est nécessaire de les inviter à mobiliser leur vécu pour chercher des éléments d'actualisation du récit et saisir la dimension universelle des thématiques. Une réflexion sur l'émotion amoureuse, travaillée par exemple en amont de la séance, devra être remobilisée. Elle favorisera la compréhension de la relation unissant Miette et Silvère.

Par ailleurs, un dossier journalistique permettant d'actualiser la problématique politique pourra être mis à leur disposition. Le recours aux images et articles de Presse, à l'œuvre cinématographique ou aux séries fictionnelles, à d'autres œuvres plus récentes, évoquant les luttes politiques d'hier à aujourd'hui sont tout aussi indispensables pour se saisir de la réflexion politique qu'impliquent les deux passages étudiés. L'ensemble de ces dossiers n'est pas nécessairement constitué par l'enseignant. Ils peuvent aussi être réalisés par les élèves eux-mêmes, dans un travail interrogeant le rôle de la Presse dans la société par exemple.

\section{Un travail sur le lexique}

Il est aussi indispensable d'engager une comparaison, par des relevés critiques d'indices, de l'expression du rapport à la fraternité au sein des deux textes, en s'appuyant sur la définition du concept. Ce sera l'occasion d'inciter les élèves à travailler le sens de la langue dans une visée critique. L'introduction du présent ouvrage permettra aisément d'apporter les connaissances nécessaires aux enseignants pour structurer cette approche. Les élèves devront saisir les enjeux de la polysémie et des glissements sémantiques progressifs d'un terme conceptuel. Une fois établie la distinction entre les deux dimensions de la fraternité, ils pourront alors se projeter avec empathie dans les états mentaux des personnages pour comprendre les jeux sociaux opposés que les deux passages mettent en évidence.

L'usage, dans la symbolique politique, des couleurs «jaune » et "rouge » sont aussi riches pour saisir la puissance évocatrice de l'œuvre de Zola. Là encore, partant du repérage sémantique de ces termes dans la lutte politique ou syndicale contemporaine, ils pourront être amenés ensuite à lire tout ou partie de l'analyse proposée par Maurice Tournier (1984) pour mieux saisir les enjeux du texte de l'auteur. Un travail d'écriture pour redéfinir l'évolution de l'usage métaphorique et métonymique avec les luttes du début du $21^{\mathrm{e}}$ siècle (par exemple autour du mouvement dit "des gilets jaunes ») pourrait clore ce travail. Il serait en effet intéressant d'observer que le jaune qui autrefois connotait l'idée de traitres, de briseurs de grèves, est, par glissement de sens, passé du côté des révoltes, prenant cette fois-ci une valeur positive et opposée.

\section{Le rôle du débat}

In fine, l'étude des textes devra déboucher sur la constitution d'un débat permettant d'interroger les actions des personnages, leurs intentions et le rapport qu'ils tissent à l'autre et à la société. Il s'agirait ici de favoriser l'émergence d'une discussion empathique et raisonnée en questionnant le rapport à la fraternité humaine illustrée 
par le récit et ressentie par les lecteurs que sont les élèves. Pour cela, il sera nécessaire de mettre en évidence les conséquences qu'impliquent de prendre la décision ou le refus de fraternité, pour les personnages de cette œuvre, et nécessairement au-delà de celle-ci.

\section{Conclusion}

Nous appuyant sur les travaux de C. Chalier et A. Bidar, nous avons interrogé quelquesunes des conditions et des limites de la transmission de la notion d'une fraternité humaine. Ces deux philosophes mettent en évidence l'essentialité des témoignages de fraternité pour lutter contre les «misères sidérantes provoquées par les ruptures du lien humain » (Chalier, 2004, p. 12). Ce faisant, ils invitent à penser les conditions de la transmission de cette valeur jugée comme fondamentale et, dans cette perspective, ouvrent une voie féconde: il est, selon eux, indispensable d'interroger les relations ipséité-altérité avec une posture critique articulant sensibilité et raisonnement. Ils postulent qu'il s'agit là d'une condition indispensable si l'on veut développer l'espoir de fraternité et prendre la décision de fraternité.

Dans un deuxième temps, grâce aux travaux des critiques et des didacticiens qui interrogent l'idée de lecture éthique de la littérature, nous avons postulé que la lecture critique de fictions littéraires pouvait apporter une contribution non négligeable à ce projet éducatif. Dans cette perspective, les didacticiens interrogent souvent les pratiques effectives ou préconisent des conduites didactiques.

Cependant, parce que l'analyse des pratiques effectives montre que les enseignants rencontrent des difficultés à interroger l'axiologie des textes (Perrin-Doucey, 2019a; Daumet, à paraitre) nous pensons qu'il est utile de les accompagner aussi dans la lecture des textes. En effet, le pilotage d'une séance implique de penser l'ensemble des éléments indispensables à la construction des dispositifs didactiques. Le format d'un article ne nous permettant pas de tout explorer, nous avons donc choisi d'interroger l'amont de la réalisation didactique. Ainsi, nous explorons le travail du professeur conduit à penser les moyens mis en œuvre dans le projet didactique : choix du corpus, analyse des textes et des outils complémentaires, délimitation des objets d'enseignement pour structurer ses séances.

\section{BIBLIOGRAPHY}

Bidar, A. (2015). Plaidoyer pour la fraternité. Paris : Albin Michel.

Chalier, C. (2004). La Fraternité, un espoir en clair-obscur. Paris : Buchet-Chastel.

Bucheton, D. (2020). Les gestes professionnels dans la classe. Paris : ESF Sciences humaines. [Édition du Kindle pour l'édition citée].

Citton, Y. (2007). Lire, interpréter, actualiser. Paris : Amsterdam. 
Compagnon, A. (2009). La littérature, pour quoi faire?, leçon inaugurale au Collège de France du 30/11/2006. Paris : Collège de France/Fayard.

Compagnon, A. (2007-2008). Cours : Morales de Proust. https://www.college-de-france.fr/media/ antoine-compagnon/UPL49209_Antoine_Compagnon_cours_0708.pdf.

Daumet, Y. (2020, à paraitre). Le moment éthique. In B. Louichon (dir.) Un texte dans la classe. Pratiques d'enseignement de la littérature au cycle 3 en France. Bruxelles : Peter Lang.

Dufays, J.-L. (2002). Les lectures littéraires : évolution et enjeux d'un concept, Tréma, 19. https:// journals.openedition.org/trema/1579.

Gritti J. (1983). Contribution sémiologique au symbole, Revue théologique de Louvain, 14, 209-214.

Jouve, V. (2001). Poétique des valeurs. Paris : Presses Universitaires de France.

Hamon, H. (1984). Texte et idéologie. Valeurs, hiérarchies et évaluations dans l'œuvre littéraire. Paris : Presses Universitaires de France.

Langlade, G. Rouxel A. (2004). Le Sujet-lecteur, lecture subjective et enseignement de la littérature. Rennes : Presses Universitaires de Rennes.

Larrivé, V. (2014). Du bon usage du bovarysme dans la classe de français : développer l'empathie fictionnelle des élèves pour les aider à lire les récits littéraires : l'exemple du journal de personnage. (Thèse de doctorat). Université Bordeaux 3.

Larrivé, V. (2015). Empathie fictionnelle et écriture en « je » fictif, Repères, 51, 155-176.

Louichon, B. et Perrin-Doucey, A. (2016). La littérature et le débat empathique et raisonnable. In M. Lebrun (dir.), Et si l'école apprenait à penser... (pp. 191-205). Bejune : HEP Bejune. Coll. « Débats ». Louichon, B. \& Sauvaire, M. (2019). Le Tournant éthique en didactique de la littérature. Repères, 58.

Mas, M. (2017). Le lecteur et le citoyen. Analyse des relations entre littérature et valeurs dans les programmes du cycle 3 de l'école primaire. Le français aujourd'hui, 197, 27-36.

Ministère de l'Education Nationale, (2015a), Onze mesures pour une grande mobilisation de l'école pour les valeurs de la république. http://eduscol.education.fr/cdi/actualites/archives/1ersemestre-2015/mesures-valeurs-rep

MEN. (2015b). L'enseignement moral et civique (EMC). Bulletin officiel spécial du 25 juin 2015. https://www.education.gouv.fr/cid90776/l-enseignement-moral-et-civique-au-bo-special-du-25juin-2015.html

Perrin-Doucey, A. (2019a). Lecture littéraire et formation morale au cycle 3 : enjeux, programmes et analyse de pratiques. Repères, 58, 81-97.

Perrin-Doucey, A. (2019b), Littérature et lecture, valeurs et citoyenneté : quels apports pour l'enseignement moral et civique ? Recherches \& Travaux, 94. http://journals.openedition.org/ recherchestravaux/1629

Rouvière, N. (2018). Enseigner La Littérature en questionnant les valeurs. Bruxelles : Peter Lang.

Rouvière, N. (2019). Les Composantes de la lecture axiologique, Repères, 58, 31-47.

Todorov, T. (2007). La Littérature en péril. Paris : Flammarion.

Tournier, M. (1984). Les jaunes : un mot-fantasme à la fin du 19e siècle. Numéro spécial : L'Autre, l'Étranger, présence et exclusion dans le discours, Mots, 8, 125-146. 
Vibert, A. (2011). Faire place au sujet lecteur en classe : quelles voies pour renouveler les approches de la lecture analytique au collège et au lycée ? https://eduscol.education.fr/lettres/im_pdflettres/ intervention-anne-vibert-lecture-vf-20-11-13.pdf

Zola, E. (1991[1871]). La Fortune des Rougon. Paris : Pocket.

\section{APPENDIXES}

\section{Annexes : extraits cités}

Extrait 1 Emile Zola, La Fortune des Rougon, ch. I, Éd. Pocket, 1991, p. 192

Quand ils furent sur le talus, dans l'ombre des broussailles, l'enfant, un peu pâle, regarda tristement ces hommes dont les chants lointains avaient suffi pour arracher Silvère de ses bras. Il lui sembla que la bande entière venait se mettre entre elle et lui. Ils étaient si heureux, quelques minutes auparavant, si étroitement unis, si seuls, si perdus dans le grand silence et les clartés discrètes de la lune ! Et maintenant Silvère, la tête tournée, ne paraissant même plus savoir qu'elle était là, n'avait de regards que pour ces inconnus qu'il appelait du nom de frères.

La bande descendait avec un élan superbe, irrésistible. Rien de plus terriblement grandiose que l'irruption de ces quelques milliers d'hommes dans la paix morte et glacée de l'horizon. La route, devenue torrent, roulait des flots vivants qui semblaient ne pas devoir s'épuiser ; toujours, au coude du chemin, se montraient de nouvelles masses noires, dont les chants enflaient de plus en plus la grande voix de cette tempête humaine. Quand les derniers bataillons apparurent, il y eut un éclat assourdissant. La Marseillaise emplit le ciel, comme soufflée par des bouches géantes dans de monstrueuses trompettes qui la jetaient, vibrante, avec des sècheresses de cuivre, à tous les coins de la vallée. Et la campagne endormie s'éveilla en sursaut ; elle frissonna tout entière, ainsi qu'un tambour que frappent les baguettes; elle retentit jusqu'aux entrailles, répétant par tous ses échos les notes ardentes du chant national. Alors ce ne fut plus seulement la bande qui chanta; des bouts de l'horizon, des rochers lointains, des pièces de terre labourées, des prairies, des bouquets d'arbres, des moindres broussailles, semblèrent sortir des voix humaines ; le large amphithéâtre qui monte de la rivière à Plassans, la cascade gigantesque sur laquelle coulaient les bleuâtres clartés de la lune, était comme couvert par un peuple invisible et innombrable acclamant les insurgés ; et, au fond des creux de la Viorne, le long des eaux rayées de mystérieux reflets d'étain fondu, il n'y avait pas un trou de ténèbres où des hommes cachés ne parussent reprendre chaque refrain avec une colère plus haute. La campagne, dans l'ébranlement de l'air et du sol, criait vengeance et liberté. Tant que la petite armée descendit la côte, le rugissement populaire roula ainsi par ondes sonores traversées de brusques éclats, secouant jusqu'aux pierres du chemin.

Extrait 2 Emile Zola, La Fortune des Rougon, Éd. Pocket, 1991, ch.3, pp. 128-129

L'idée de réussir, de voir toute sa famille arriver à la fortune, était devenue une monomanie chez Félicité. Pascal, pour ne pas la chagriner, vint donc passer quelques soirées dans le salon jaune. Il s'y ennuya moins qu'il ne le craignait. La première fois, il fut stupéfait du degré d'imbécillité auquel un homme bien portant peut descendre. Les 
anciens marchands d'huile et d'amandes, le marquis et le commandant eux-mêmes, lui parurent des animaux curieux qu'il n'avait pas eu jusque-là l'occasion d'étudier. Il regarda avec l'intérêt d'un naturaliste leurs masques figés dans une grimace, où il retrouvait leurs occupations et leurs appétits ; il écouta leurs bavardages vides, comme il aurait cherché à surprendre le sens du miaulement d'un chat ou de l'aboiement d'un chien. À cette époque, il s'occupait beaucoup d'histoire naturelle comparée, ramenant à la race humaine les observations qu'il lui était permis de faire sur la façon dont l'hérédité se comporte chez les animaux. Aussi, en se trouvant dans le salon jaune, s'amusa-t-il à se croire tombé dans une ménagerie. Il établit des ressemblances entre chacun de ces grotesques et quelque animal de sa connaissance. Le marquis lui rappela exactement une grande sauterelle verte, avec sa maigreur, sa tête mince et futée. Vuillet lui fit l'impression blême et visqueuse d'un crapaud. Il fut plus doux pour Roudier, un mouton gras, et pour le commandant, un vieux dogue édenté. Mais son continuel étonnement était le prodigieux Granoux. Il passa toute une soirée à mesurer son angle facial. Quand il l'écoutait bégayer quelque vague injure contre les républicains, ces buveurs de sang, il s'attendait toujours à l'entendre geindre comme un veau; et il ne pouvait le voir se lever, sans s'imaginer qu'il allait se mettre à quatre pattes pour sortir du salon.

- Cause donc, lui disait tout bas sa mère, tâche d'avoir la clientèle de ces messieurs. - Je ne suis pas vétérinaire, répondit-il enfin, poussé à bout.

\section{NOTES}

1. Cependant l'acception juridique parait s'imposer aujourd'hui puisqu'en 2018, le conseil constitutionnel a introduit le "principe de fraternité » pour éliminer du champ des poursuites possibles les aides apportées aux migrants en situation de détresse.

2. L'expression tend à montrer que, comme toujours chez Zola, la restitution imaginale d'une scène forte en émotion synthétise plusieurs sensations, ce qui lui donne cette puissance synesthésique singulière. Dans le texte, l'auteur donne en effet à voir (scène épique théâtralisée) et à entendre (la nature répercute à tous les échos la Marseillaise entonnée par les insurgés) ce moment clé de la révolte populaire.

3. Dans son poème intitulé «Les Assis », Rimbaud stigmatisme en une vision monstrueuse et avec une ironie féroce la domination des petits bourgeois bien-pensants (ici des bibliothécaires). Rivés à leurs ronds de cuir, immobiles et " genoux aux dents ", ils sont de redoutables gardiens du conservatisme social et politique, avec « une main invisible et qui tue » si on les dérange, comme les habitués du salon jaune des Rougon.

4. Sur l'acception métaphorique et son usage en 1871, voir l'étude de Maurice Tournier (1984) qui lui est consacrée.

\section{ABSTRACTS}

The article questions, in support of the work of C. Chalier and A. Bidar, the notion of fraternity in its twofold philosophical and humanistic dimension. Insofar as, in the aftermath of the attacks of 
2015 and 2016, the French Ministry of National Education invites teachers to develop hope and the decision of fraternity, the authors elaborate a reflection inscribed in the literary field to highlight certain conditions of this transmission. Drawing on the recent work of some critics and literary educators, they then plan to anchor this teaching in a dynamic that includes literary fiction, history and artistic culture. To do this, they propose to carry out a comparative reading with a didactic vocation of two extracts from Zola's novel La Fortune des Rougon, thought to be fruitful supports for designing a project of teaching literature through values.

L'article interroge, à l'appui des travaux de C. Chalier et A. Bidar, la notion de fraternité dans sa double dimension philosophique et humaniste. Dans la mesure où, aux lendemains des attentats de 2015 et 2016, le Ministère de l'Education Nationale français invite les enseignants à développer l'espoir et la décision de fraternité, les auteurs élaborent une réflexion inscrite dans le champ littéraire pour mettre en évidence certaines conditions de cette transmission. S'appuyant sur les travaux récents de quelques critiques et des didacticiens de la littérature, ils prévoient ensuite d'ancrer cet enseignement dans une dynamique incluant la fiction littéraire, l'histoire et la culture artistique. Pour ce faire, ils se proposent de réaliser une lecture comparée à vocation didactique de deux extraits du roman de Zola La Fortune des Rougon, pensés comme des supports féconds pour concevoir un dispositif d'enseignement de la littérature par les valeurs.

INDEX

Keywords: fraternity, education, literature, didactics, values, citizenship

Mots-clés: fraternité, éducation, littérature, didactique, valeurs, citoyenneté

\section{AUTHORS}

AGNÈS PERRIN-DOUCEY

Université de Montpellier, LIRDEF

JACQUES-LOUIS PERRIN

Lycée Camille Vernet 